\title{
Wnt5a regulates Ameloblastoma Cell Migration by modulating Mitochondrial and Cytoskeletal Dynamics
}

\author{
Xue Qiao ${ }^{3,4^{*}}$, Xing $\mathrm{Niu}^{1 *}$, Junxiu Shi ${ }^{5}$, Lijie Chen ${ }^{1}$, Xiaobin Wang ${ }^{6}$, Jinwen Liu , Li Zhu ${ }^{3}$, Ming Zhong ${ }^{1,2}$ \\ 1. Department of Oral Histopathology, School and Hospital of Stomatology, China Medical University, Liaoning Province Key Laboratory of Oral Disease, \\ Shenyang, Liaoning, China. \\ 2. Department of Stomatology, Xiang' an Hospital of Xiamen University, Xiamen, Fujian, China. \\ 3. Department of Central Laboratory, School and Hospital of Stomatology, China Medical University, Liaoning Province Key Laboratory of Oral Disease \\ Shenyang, Liaoning, China. \\ 4. Department of Oral Biology, School and Hospital of Stomatology, China Medical University, Liaoning Province Key Laboratory of Oral Disease, Shenyang, \\ Liaoning, China \\ 5. Department of Developmental Cell Biology, Cell Biology Division, Key Laboratory of Cell Biology, Ministry of Public Health, Key Laboratory of Medical \\ Cell Biology, Ministry of Education, China Medical University, Shenyang, Liaoning, China. \\ 6. Department of Orthodontics, School and Hospital of Stomatology, China Medical University, Liaoning Province Key Laboratory of Oral Disease, Shenyang, \\ Liaoning, China. \\ 7. Department of Periodontics, School and Hospital of Stomatology, China Medical University, Liaoning Province Key Laboratory of Oral Disease, Shenyang, \\ Liaoning, China.
}

*These authors contributed equally to this work.

$\triangle$ Corresponding author: Ming Zhong, E-mail: mzhong@cmu.edu.cn; Department of Oral Histopathology, School and Hospital of Stomatology, China Medical University, Liaoning Province Key Laboratory of Oral Disease, No.117 North Nanjing Street, Shenyang 110002, Liaoning, China. Department of Stomatology, Xiang' an Hospital of Xiamen University, No.2000 Xiang' an East Road, Xiang'an District, Xiamen 361102, Fujian, China.

(c) The author(s). This is an open access article distributed under the terms of the Creative Commons Attribution License (https://creativecommons.org/licenses/by/4.0/). See http://ivyspring.com/terms for full terms and conditions.

Received: 2020.04.01; Accepted: 2020.06.30; Published: 2020.07.11

\begin{abstract}
Objective: Abnormal expression of $\mathrm{Wnt5a}$ has been detected in various tumors, including ameloblastoma $(A B)$. Yet, there is no specific mechanistic evidence for the functional role of Wnt5a in $A B$. In this study, we aimed to conduct a mechanistic examination of the importance of $W n t 5 a$ in $A B$ development.

Methods: The expressions of $\mathrm{Wnt5a}$ and CorolA were examined by Western blot and immunohistochemistry both in $A B$ tissues and $A M-1$ cells. The number and size of mitochondria were detected by electronic transmission microscope and confocal microscope. Gain-of-function and loss-of-function assays were used to explore the biological roles of $\mathrm{Wnt5a}$ and CorolA in organelle dynamics changes and cell migration. Cell migration was detected by wound healing and transwell assay.

Results: We found that in AM-1 cells, up-regulation of Wnt5a led to enhanced mitochondrial energy production and altered calcium homeostasis, with elevated calcium levels directly leading to altered mitochondrial dynamics and interactions between the cytoskeleton and the mitochondria. When Wnt5a or its downstream cytoskeleton-associated protein CorolA was knocked down, the migration capacity of AM-1 cells was markedly impaired.

Conclusion: Together, these results suggest that $\mathrm{Wnt5}$ a plays mitochondria and cytoskeleton specific roles in regulating the development of human $A B$, with its down-regulation leading to impaired tumor development, thus highlighting $\mathrm{Wnt5a}$ or CorolA as potentially viable therapeutic targets for the treatment of $A B$.
\end{abstract}

Key words: ameloblastoma; mitochondria-cytoskeleton; Wnt5a; Coro1A; migration

\section{Introduction}

Ameloblastoma (AB) is a common epithelial tumor, accounting for more than $60 \%$ of odontogenic tumors [1, 2]. AB is typically composed of enamel-like structures without any mature enamel or hard tissue being present. According to the latest WHO Classification of Head and Neck Tumors, ABs are 
highly diverse with four primary pathological subtypes being recognized: $A B$, unicystic, and extraosseous/peripheral types [3]. These tumors typically arise in the jaw, driving localized swelling and deformities of the face [4]. Typical treatment of ABs entails radical jaw excision, but the resultant facial deformities could have a markedly adverse impact on the physical and mental health of treated patients. However, when patients instead undergo more conservative treatment, recurrence is common, in some cases leading to malignant transformation and metastasis [5-7]. As such, it is vital that molecular therapeutic targets are identified to guide $A B$ treatment so as to ensure that patients have satisfactory clinical outcomes.

Mitochondria are essential intracellular organelles both for regulating energy production within cells, and for buffering intracellular $\mathrm{Ca}^{2+}$ levels and mediating interactions between organelles. It is also well known that they are closely linked with the development of tumors, with mitochondrial damage in tumor cells disrupting the normal balance between oxidative phosphorylation and glycolysis, thereby resulting in characteristic metabolic reorganization that is frequently observed in tumors $[8,9]$. The number, morphology, and localization of mitochondria within cells are highly variable, and are closely related to the invasive and migratory capabilities of tumor cells [10,11]. The cytoskeleton can also regulate mitochondrial intracellular dynamics. Some studies suggest that actin-related proteins regulate mitochondrial fission and contact between mitochondria and the cytoskeleton [12]. Remodeling of the cytoskeleton and mitochondrial network can have a profound impact on the motility of cells, and is thus a key component of tumor progression [13]. However, to date, no studies have specifically examined the changes in mitochondrial dynamics or organelle interactions that occur during $\mathrm{AB}$ development, with the underlying molecular mechanisms therefore being wholly uncharacterized.

Proteins in the Wnt family facilitate paracrine and autocrine activation of specific cell membrane receptors [14]. Wnt5a can regulate cellular signaling through non-canonical Wnt signaling pathways, with reported roles in the development and progression of various tumor types [15], including elevated Wnt5a expression in oral squamous cell carcinoma, tongue cancer and ameloblastoma [16-18]. Wnt family proteins have been reported to play key roles in regulation of mitochondrial quality control and energy metabolism. For example, Wnt3a overexpression mediates enhanced mitochondrial basal oxygen consumption and up-regulates proteins associated with oxidative phosphorylation [19].
Classical Wnt/ $\beta$-catenin signaling can, in concert with PTEN signaling, additionally mediate the enhanced fusion of damaged mitochondria and inhibit mitophagy, resulting in altered mitochondrial remodeling, abnormal mitochondrial accumulation, and altered cellular migration and motility [20]. How the non-canonical Wnt5a/ $\mathrm{Ca}^{2+}$ signaling pathway regulates mitochondrial network dynamics and organelle interactions within cells, however, is not as well understood.

In the present study, we aimed to expound the specific mechanistic evidence for the functional role of up-regulated Wnt5a in $\mathrm{AB}$. Its overexpression led to significant increases in mitochondrial and intracellular calcium, resulting in substantial mitochondrial and cytoskeletal remodeling. When Wnt5a or its downstream cytoskeleton associated target protein Coro1A were knocked down, this significantly ablated these changes in intracellular organelle dynamics and suppressed the migratory activity of $\mathrm{AB}$ cells. At present, there is a lack of relevant research on the role of Coro1A in $A B$. In summary, these findings offer a novel insight into the function of Wnt5a in the regulation of organelle dynamics and $\mathrm{AB}$ progression, potentially highlighting this protein and its associated pathway as viable targets for $\mathrm{AB}$ therapy.

\section{Materials and Methods}

\section{Tissue specimens}

In total, 15 paired $\mathrm{AB}$ and adjacent normal tissue specimens were obtained from patients that underwent surgical tumor resection at the Department of Oral and Maxillofacial Surgery in the School of Stomatology of China Medical University from 2016 to 2018. Upon collection, tissues were immediately stored at $-80{ }^{\circ} \mathrm{C}$ prior to downstream analysis. We also collected 91 AB immunohistochemical sections from the School and Hospital of Stomatology, China Medical University between January 2015 to March 2016. Moreover, 20 cases of normal oral mucosa (NOM) tissues were obtained from patients undergoing surgical removal of the third mandibular molar. The study was approved by the Ethics Committee of School and Hospital of Stomatology, China Medical University (2016-12), and all patients signed informed consent.

\section{Cell culture}

AM-1 cells used in the present study were generously gifted by Professor Hidemitsu Harada (School of Dentistry, Iwate Medical University, Japan), and were grown between passage 15 and 25 on the Keratinocyte-SFM (Gibco, Invitrogen, USA). Human keratinocytes (HaCaT cells, ATCC, USA) 
from passage 5-17 were cultured in DMEM (Gibco, Invitrogen, USA) plus 10\% FBS and penicillin/ streptomycin.

\section{Immunohistochemistry}

After paraffin embedding, $4 \mu \mathrm{m}$ thick sections of $A B$ tissue samples were prepared. These sections were deparaffinized by xylene, rehydrated by an ethanol gradient, and quenched by endogenous peroxidase activity via treatment for $30 \mathrm{~min}$ in methanol containing $0.3 \% \quad \mathrm{H}_{2} \mathrm{O}_{2}$. Samples were then microwaved in citrate phosphate buffer $(\mathrm{pH}$ 6.0) to mediate antigen retrieval. Then, samples were incubated with polyclonal rabbit anti-Wnt5a (1:2000, ab229200, Abcam, MA, USA) at $4{ }^{\circ} \mathrm{C}$ overnight, followed by secondary anti-rabbit IgG (1:5000, Santa Cruz Biotechnology, USA) at room temperature for 2 h.

Staining results were scored based upon both (A) staining intensity (with $0,1,2$, and 3 corresponding to negative, weak, moderate, and strong staining, respectively), and (B) Wnt5a-positive tumor cells frequency (with $1,2,3$, and 5 corresponding to $0-10 \%$, $11-50 \%, 51-80 \%$, and $81-100 \%$, respectively) [21]. These two scores were then multiplied together, yielding a final staining score. When scores $>3$, the samples were considered Wnt5a-positive, otherwise they were considered Wnt5a-negative.

\section{Western blot}

After protein extraction from the indicated samples, equivalent amounts $(40-80 \mu \mathrm{g})$ of protein from each sample was separated via $10 \%$ SDS-PAGE and transferred onto PVDF membranes. These blots were then blocked with 5\% non-fat milk and incubated at $4{ }^{\circ} \mathrm{C}$ overnight with the following antibodies: anti-Wnt5a (1:2000, ab229200, Abcam), anti-GAPDH (1:2500, ab9485, Abcam), anti-Coro1A (1:2000, ab72212, Abcam). GAPDH was used as an internal control. Blots were then incubated with secondary goat anti-rabbit IgG (1:10000, A21020, Abbkine) for 1h. Finally, Odyssey imaging system was used for protein visualization.

\section{Confocal microscopy}

FITC-phalloidin $(0.2 \mu \mathrm{M}, 30 \mathrm{~min})$ and MitoTracker Red $(0.2 \mu \mathrm{M}, 10 \mathrm{~min})$ (Invitrogen, USA) were used to treat AM-1 cells, after which a Nikon A1+ confocal microscope equipped with a 40X 1.3NA oil immersion objective was used for the confocal imaging of these stained tumor cells. Excitation at 488 $\mathrm{nm}$ and $562 \mathrm{~nm}$ was used to mediate dual excitation of these two fluorophores, with respective emission being detected at 505-530 $\mathrm{nm}$ and > $560 \mathrm{~nm}$, respectively.

\section{Assessment of mitochondrial respiration}

A Seahorse XF24 Extracellular Flux Analyzer (Seahorse Bioscience, USA) was used to assess mitochondrial respiration based on provided directions. Briefly, AM-1 cells were added to XF microplates (10,000 cells/well), after which cellular OCR was assessed in assay medium (Sigma, USA) to which oligomycin $(1 \mu \mathrm{M}), \mathrm{FCCP}(500 \mathrm{nM})$, rotenone (1 $\mu \mathrm{M})$, and antimycin $\mathrm{A}(1 \mu \mathrm{M})$ were added in sequence.

\section{Intracellular calcium imaging}

Initially, $5 \times 10^{4} \mathrm{AM}-1$ cells were plated on $25 \mathrm{~mm}$ coverslips and were treated for $30 \mathrm{~min}$ with $1 \mu \mathrm{M}$ Fluo-4 (Molecular Probes, Invitrogen, USA) at $37^{\circ} \mathrm{C}$. Cells were then washed thrice with KSFM media followed by confocal imaging with a Nikon A1+ confocal microscope (Nikon, Japan). Cells were imaged with excitation and emission wavelengths of $488 \mathrm{~nm}$ and 505-530 nm, respectively, with bidirectional scanning mode being used to acquire 400 $128 \times 128$ pixel frames $(0.244 \mathrm{~s} /$ frame). The Wnt5acontaining media was then added to cells, and cell images were recorded for $5 \mathrm{~min}$, with fluorescence intensity being reported as $\Delta \mathrm{F} / \mathrm{F}_{0}$ as indicated by $\left(\mathrm{F}-\mathrm{F}_{\text {base }}\right) / \mathrm{F}_{\text {base }}$.

\section{Cell migration assay}

The migration of AM-1 cells was assessed using wound healing and transwell assays. For the former, a monolayer of AM-1 cells $(80 \%$ confluent $)$ was scratched using a $100 \mu 1$ pipette tip, after which this wound was washed thrice using PBS and the migration distance of wound edge was calculated with Image J software. In the transwell assays, AM-1 cells in $100 \mu \mathrm{l}$ serum-free media were added to the upper chamber of a 24-well plate (Costar, USA). After $24 \mathrm{~h}$, the cells that had migrated to the lower chamber of this plate were fixed using ethanol and stained with hematoxylin and eosin.

\section{Recombinant adenovirus preparation}

Double Wnt5a-specific siRNA sequences (5'-GA AGCCCAUUGGAAUAUUAUU-3' and 5'-GCUGGA AGUGCAAUGUCUUCC-3') were designed based upon the Wnt5a gene sequence and were synthesized by Genepharma (Shanghai, China). A non-targeted siRNA sequence 5'-UUUGCACUGUGCAAGCCUC UU-3' was used as a negative control. These sequences were inserted into the pENTR plasmid recombined with pBLOCK-iT ${ }^{\mathrm{TM}}$ 6-DEST vector and an Adenoviral Expression System (Invitrogen) was used to prepare adenoviral particles that were purified with a Vivapure ${ }^{\circledR} A d e n o P A C K T M 20 R T$ Kit (Sartorius). A Coro1A-specific siRNA (5'-UUGUCUACUCGUCCA 
GUCUUGCCUA-3') was also designed and synthesized. These sequences were then used to prepare into adenoviral particles as above.

\section{Reagents}

Recombinant human Wnt5a protein was purchased from R\&D systems; anti-Wnt5a was from Abcam; anti-Coro1A and anti-NFATc2 were from Affinity; anti-Lamin B1 was from Origene; Mitotracker Red, phalloidin FITC, and fluo-4 were from Molecular Probes.

\section{Statistical analysis}

Data are presented as means \pm SEM. Each experiment was repeated at least three times. The difference was compared via unpaired Student's t-test or one-way ANOVA with Bonferroni's post hoc test as appropriate. In addition, correlations between the Coro1A expression and clinical characteristics of $\mathrm{AB}$ patients were assessed by Chi-square test. GraphPad Prism 5.0 (Graph Pad Software, Inc., San Diego, CA, USA) was used for all statistical analyses, with $P<0.05$ as the significant threshold.

\section{Results}

\section{AB tissues exhibit elevated $\mathbf{W n t 5}$ a expression}

We firstly explored the role of Wnt5a in $A B$ by comparing its immunohistochemical expression in 91 $\mathrm{AB}$ tissues and 20 normal tissues (Figure 1A). Consistent with previously studies by Sukarawan et al. [18], we found that Wnt5a expression was clearly evident in $A B$ samples, and the positive expression rate in these tumor tissues was significantly higher than normal control samples (Table 1). This result was further supported by Western blot, which additionally confirmed elevated Wnt5a protein expression in $\mathrm{AB}$ tissues (Figure 1B). Furthermore, we observed about 2-fold increase in Wnt5a expression in an $A B$ cell line (AM-1 cell line) relative to levels in control HaCaT cells (Figure 1C). Together, these data suggest a possible involvement of Wnt5a in the process of $\mathrm{AB}$ development.

\section{Wnt5a regulates energy synthesis by modulating mitochondrial calcium homeostasis}

Previous research has highlighted a role for secreted Wnt5a in mediating signaling that ultimately leads to the opening of endoplasmic reticulum $\mathrm{IP}_{3} \mathrm{R}$, resulting in $\mathrm{Ca}^{2+}$ release into the cytoplasm $[22,23]$. However, in contrast to these reports, work from Koopmans et al. suggests that Wnt5a does not play a significant role in the regulation of calcium homeostasis in the cytoplasm [24]. As such, further research was warranted to understand whether
Wnt5a plays a role in regulating mitochondrial calcium homeostasis in $\mathrm{AB}$ cells, and if so what signaling pathways are involved in such regulatory activity. To that end, we assessed cytoplasmic and mitochondrial calcium and ATP levels in the human AM-1 cells following treatment with recombinant Wnt5a. These results revealed the ability of Wnt5a to both drive mitochondrial energy production and to modulate intracellular calcium homeostasis within these cells, with intracellular and mitochondrial peak calcium levels rising by $2.02 \pm 0.16$ folds and $1.41 \pm$ 0.11 folds following Wnt5a treatment, respectively, relative to levels in control cells (Figure 2A, B). Treatment with Wnt5a further led to elevated basal respiration-associated OCR and ATP productionassociated OCR (Figure 2C). When we treated cells with sFRP5, a well-recognized Wnt signaling pathway antagonist, to inhibit Wnt5a, both the cytoplasmic and mitochondria calcium increase had greatly dropped and no statistically significant increase was observed in basal respiration and ATP production associated OCR compared with control cells (Figure 2). Together, these findings thus suggest that in $A B$ cells, Wnt5a can induce slight changes in calcium homeostasis that in turn alter mitochondrial function within these cells.

\section{Wnt5a modulates mitochondrial dynamics in ameloblastoma}

In addition to regulate mitochondrial energy production, changes in calcium homeostasis are also closely linked to both cellular survival and mitochondrial morphology [25]. We therefore utilized transmission electron microscopy (TEM) to assess mitochondrial morphology in $\mathrm{AB}$ tumor tissues and healthy control tissues. We found that relative to normal control samples, the numbers of mitochondria were significantly increased from $40.0 \pm 2.06$ to $62.7 \pm$ 5.48 per $100 \mu^{2}$ but with smaller size in either total mitochondria area percentage or single mitochondria area in $\mathrm{AB}$ samples (Figure $\mathbf{3 A}$ ), while the observed mitochondria dynamic changes suggested that these altered mitochondrial dynamics were closely associated with the onset and progression of $\mathrm{AB}$. To modulate Wnt5a protein levels, we next treated AM-1 cells with either recombinant Wnt5a to exogenously up-regulate or with an knockdown adenovirus to down-regulate Wnt5a expression. Wnt5a protein expression was reduced to about $30 \%$ of control group after knockdown adenovirus transfection through western blot (Figure 3B). These gain- and loss-of-function approaches were then used to assess the influence of Wnt5a on mitochondrial fission and fusion within these $A B$ cells. Through confocal imaging, it was determined that excess Wnt5a 
resulted in decrease of mitochondrial length, whereas its knockdown had an opposite effect (Figure 3C). Consistent with our TEM findings from $A B$ tissues, we additionally found that treatment of AM-1 cells with exogenous Wnt5a was associated with mitochondria dynamic changes (Figure 3D). Collectively, our data suggest that both in AB cells or tumor tissues, Wnt5a can regulate dynamics changes of mitochondria.

\section{Wnt5a modulates mitochondrial-cytoskeletal colocalization via regulating Coronin-1 a expression}

Through the use of ultra-high resolution microscopy, it is possible to monitor dynamic changes in inter-organelle contacts and interactions, which play key roles in regulating myriad cellular processes.
Importantly for the present study, mitochondria can interact with the cytoskeleton such that mitochondria serve as an energy source that can facilitate cytoskeletal remodeling, while the cytoskeleton itself can regulate changes in mitochondrial dynamics [26, 27]. Interplay between these organelles can thus regulate cellular energy availability, intracellular transport, and cell motility [28, 29]. In the present study, we found that Wnt5a could increase co-localization between mitochondria and the cytoskeleton with the Pearson correlation coefficient risen from $1.0 \pm 0.13$ to $1.47 \pm 0.16$ (Figure $4 \mathrm{~A}$ ), thereby modulating cellular morphology and suggesting that the regulation of such contacts may be a key mechanism whereby Wnt5a regulates the behavior of $\mathrm{AB}$ cells.

A

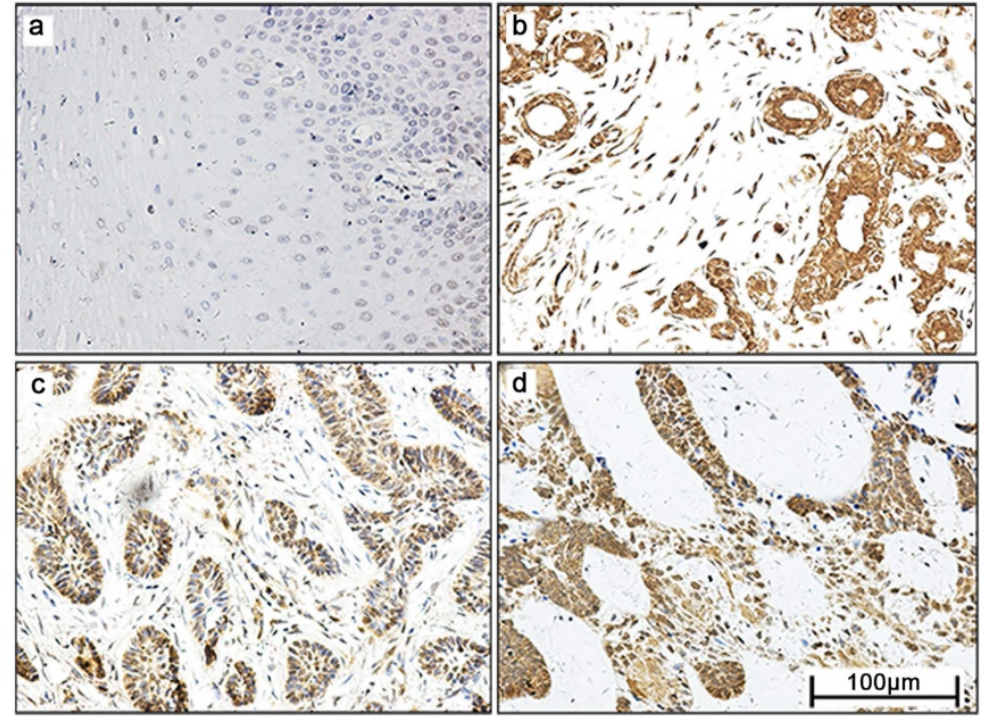

B

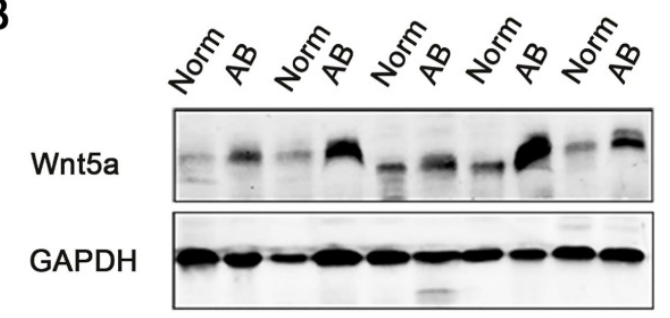

C
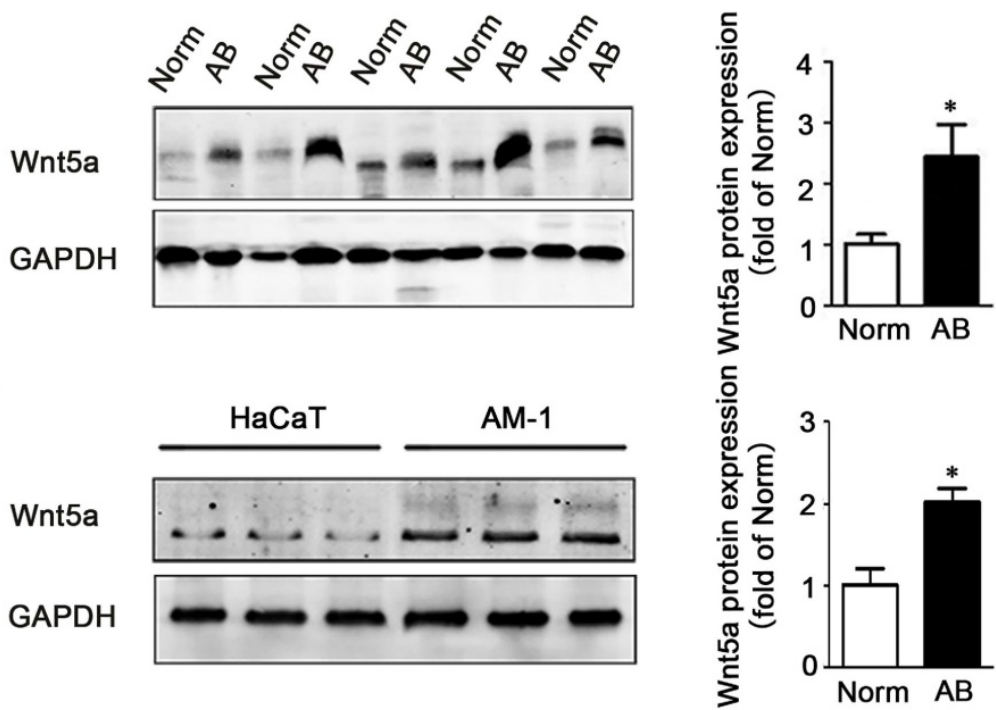

Figure 1. Expression of Wnt5a in ameloblastoma tissues and AM-1 cells. (A) Immunohistochemistry staining demonstrating Wnt5a protein expression in normal oral mucosa tissues (a), ameloblastoma tissues (b-d) (forlicular type for d\&c, plexiform type for d). Scale Bar: $100 \mu$ m. Magnification: 200x. (B) Western blot showed Wnt5a protein expression in tumor adjacent normal and ameloblastoma tissues. (C) Western blot showed Wnt5a protein expression in HaCaT and AM-1 cell lines. *p $<0.05$. 
Table 1. The differences of Wnt5a expression between ameloblastoma and normal oral mucosal tissues

\begin{tabular}{lllll}
\hline Groups & Total $(\mathrm{N})$ & Wnt5a Positive $(\mathrm{N})$ & Wnt5a Negative $(\mathrm{N})$ & $\mathrm{P}$ \\
\hline Ameloblastoma & 91 & 78 & $\chi^{2}$ & 13 \\
Normal oral mucosal tissue & 20 & 2 & 18 & 46.70 \\
\hline
\end{tabular}

P value was determined by Chi-square test. ${ }^{*} \mathrm{P}<0.05$.

A
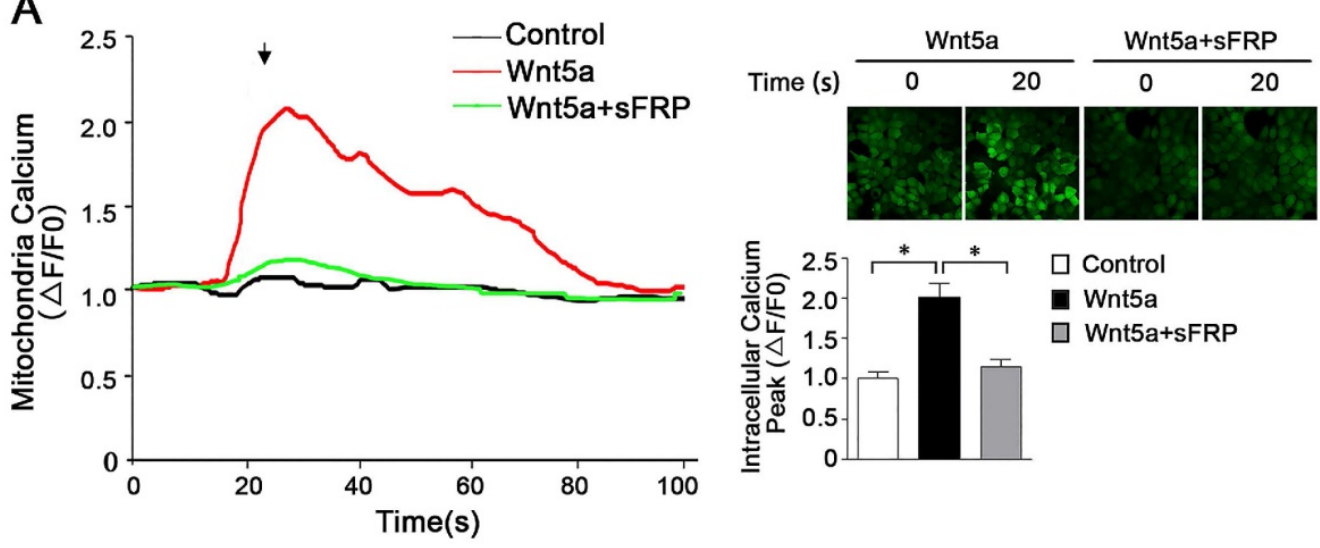

B
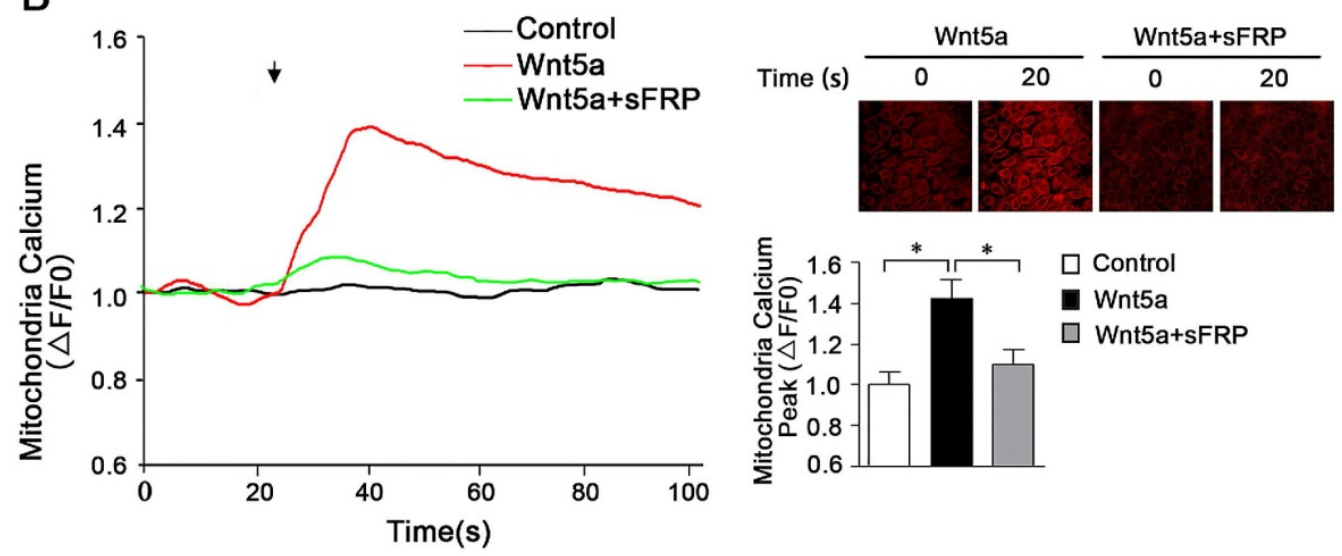

C
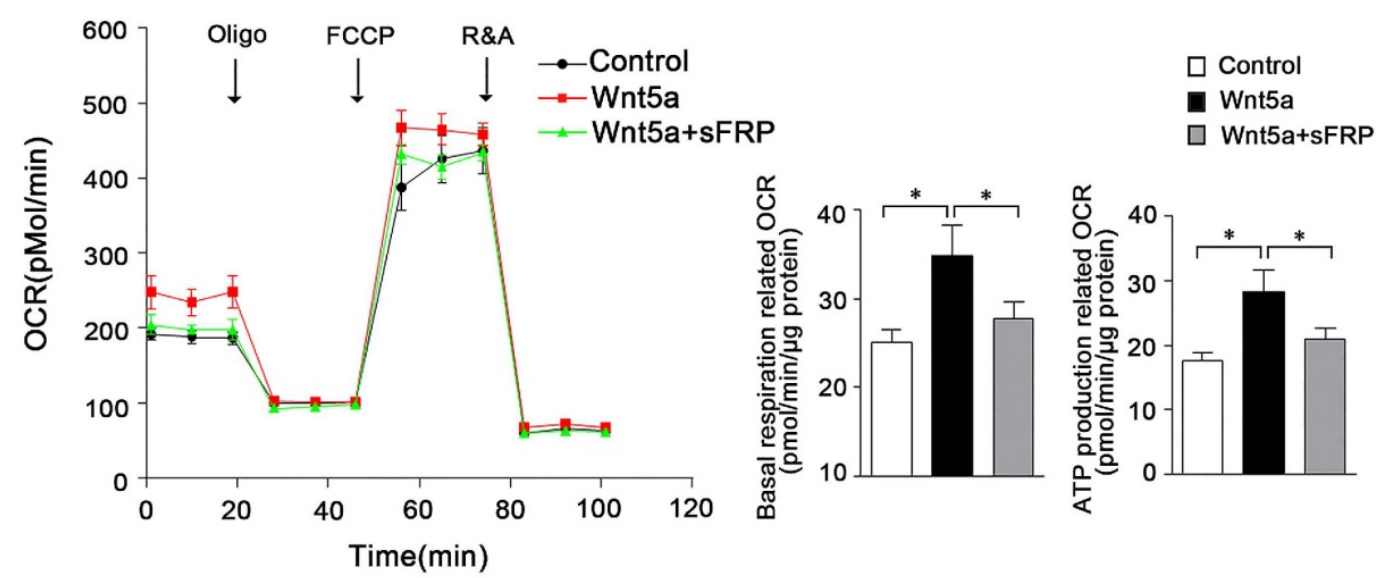

Figure 2. Wnt5a regulates energy synthesis through manipulating mitochondria calcium hemostasis. (A) Confocal images and representative recordings of cytosolic $\mathrm{Ca}^{2+}$ in Fluo-4-AM loaded AM-1 cells. The graph showing intracellular calcium changes induced by Wnt5a (200 nm) recombinant protein or Wnt5a plus sFRP5 protein addition. $\mathrm{N}=30$ cells from 4 independent experiments. Bar chart represented quantification of peak amplitude. (B) Confocal images and representative recordings of mitochondrial $\mathrm{Ca}^{2+}$ in Rhod-2-AM loaded AM-1 cells. The graph showing mitochondria calcium change after Wnt5a or Wnt5a plus sFRP5 recombinant protein addition. $\mathrm{N}=32-35$ cells from 4 independent experiments. Bar chart represented quantification of peak amplitude. (C) Traces of oxygen consumption rate (OCR) of AM-1 cells treated with Wnt5a or Wnt5a plus sFRP5, as measured with the XF24 metabolic analyzer by sequential, in port additions of mitochondrial effectors at time points indicated by downward arrows. Bar charts illustrating basal respiration related OCR and ATP production related OCR, $n=10$ from 3 independent experiments. * $\mathrm{p}^{\circ} 0.05$. 
A
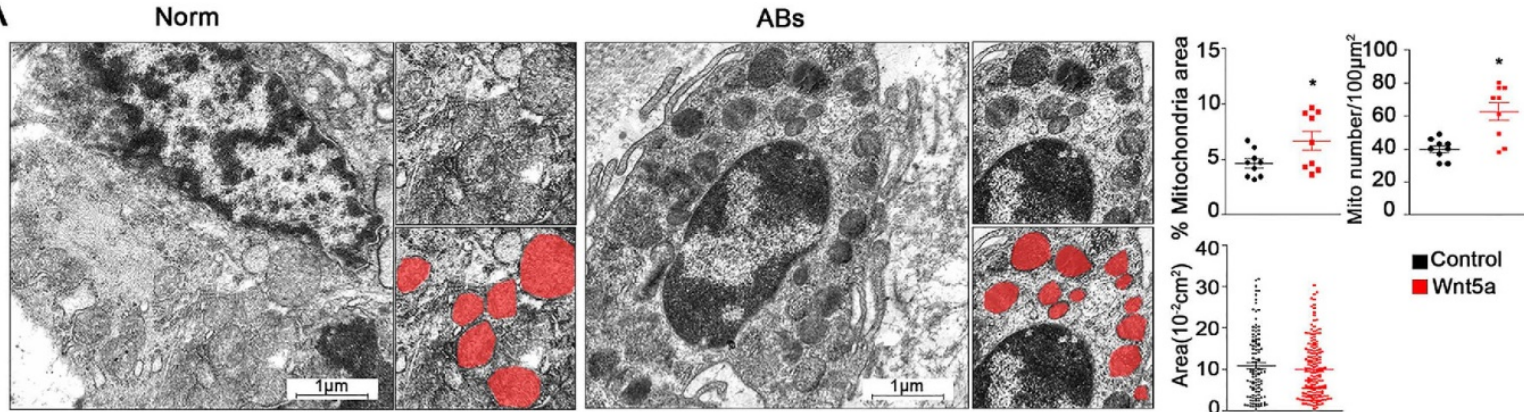

B
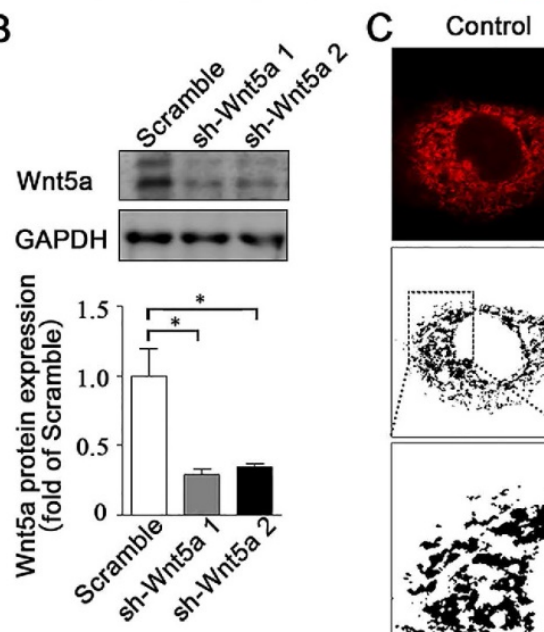

Wnt5a

scramble

sh-Wnt5a
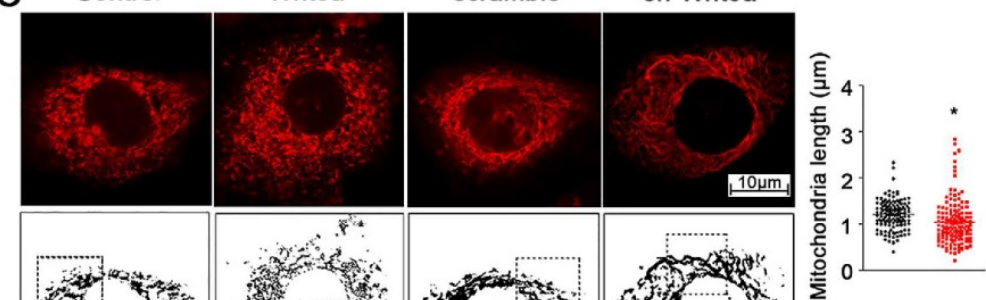

- Contro

Wnt5a
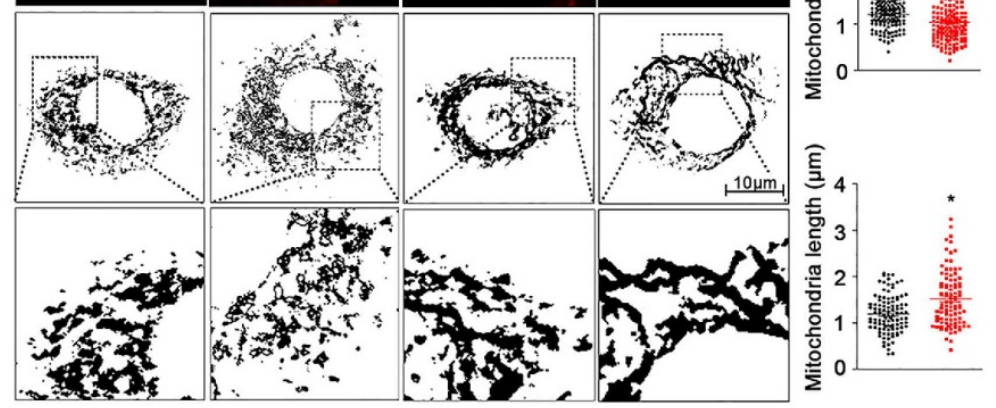

ascramble

ash-Wnt5a

\section{D}
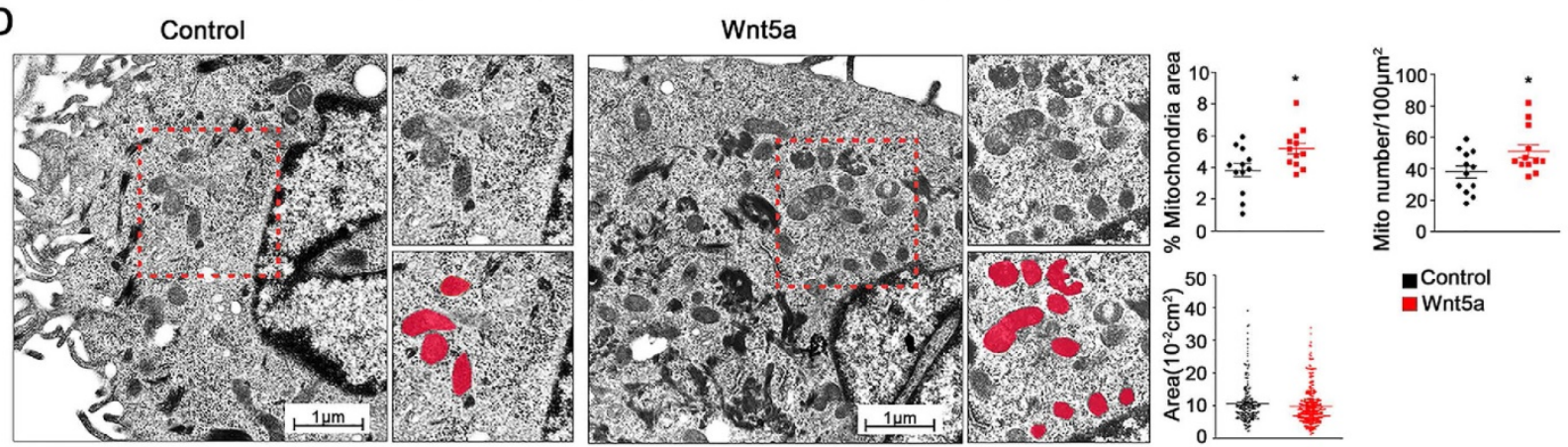

Figure 3. Wnt5a regulates mitochondria dynamics and morphology. (A) Micrographs from transmission electron microscope showing representative mitochondria morphology and number of tumor adjacent normal tissues and ameloblastoma tissues. Corresponding quantification plot charts demonstrating percentage mitochondria area fraction, mitochondria number per $100 \mu \mathrm{m}^{2}$ and average area of single mitochondria. $\mathrm{N}=9$ for area and number, $\mathrm{N}=114,116$ independent mitochondria from 3 independent experiments for single mitochondria. (B) Western blot detecting protein expression manipulated by Wnt5a shRNA adenovirus. (C) Confocal images showing representative mitochondria morphology of AM-1 cells treated with Wnt5a recombinant protein or Wnt5a knockdown adenovirus. Scatter plot charts showed quantitative analysis of mitochondria length. $\mathrm{N}=126,145$ for $\mathrm{Wnt} 5 \mathrm{a}$ up-regulation and $\mathrm{N}=101,94$ for Wnt5a knockdown from 3 independent experiments. (D) TEM images demonstrated mitochondria number and size of AM-1 cells with or without Wnt5a protein addition. Corresponding quantification demonstrate percentage mitochondria area fraction, mitochondria number per $100 \mu \mathrm{m}^{2}$ and single mitochondria area. $\mathrm{n}=12$ for mitochondria area fraction and number, $\mathrm{N}=222,302$ for independent mitochondria from 4 independent experiments. $* \mathrm{p}<0.05$.

How precisely Wnt5a regulates mitochondrial functionality remains unclear, and understanding of how interactions between mitochondria and the cytoskeleton are regulated is likewise limited in the context of controlling cellular motility and migration. To further explore how Wnt5a regulates mitochondrial-cytoskeleton interactions, we therefore next conducted gene chip analysis using two pairs of control and recombinant Wnt5a cultivation AM-1 cells (not shown). The results reflected a cytoskeleton binding protein Coronin-1A (Coro1A) with the highest different expression between normal and
Wnt5a up-regulated cells. Western blotting confirmed that, relative to control cells, Wnt5a cultivating cells contained $1.65 \pm 0.14$ folds higher levels of the Coro1A protein (Figure 5A). Moreover, Western blot results also showed that more NFAT1 (NFATc2) protein transferred to nuclear (Figure 5A). When NFATc2 nuclear translocation inhibitor, FK506, was used to treat Wnt5a cultivating cells, the up-regulation of Coro1A expression induced by Wnt5a was successfully blocked (Figure 5B). These results together suggested that such up-regulation of Coro1A may be linked with elevated calcium levels and 
consequent activation of the NFAT signaling pathway caused by Wnt5a up-regulation. Also, we observed higher Coro1A expression in AM-1 cells relative to levels in control HaCaT cells (Figure 5C). We then next assessed Coro1A expression levels via western blotting and immunohistochemistry in $91 \mathrm{AB}$ tissue samples and 20 normal tissue samples (Figure 5D, E). These analyses revealed significantly higher rates of Coro1A positivity in $\mathrm{AB}$ samples relative to controls (not shown), with this high expression being closely linked to tumor recurrence rate (Table 2). Collectively, our data suggest that Wnt5a regulates the mitochondria and cytoskeleton contacts and also up-regulates F-actin binding protein Coro1A expression through activating NFAT signaling pathway.

\section{Wnt5a-mediated regulation of Corol A promotes ameloblastoma migration and morphological change}

The ability of tumor cells to migrate within an organism is a hallmark of these cells, and is also linked to both ATP production and mitochondrial dynamics $[11,30]$. As mitochondria move along the cytoskeleton, they can serve as an energy source to support further reorganization of organelles within the cell [26]. As such, close contact between the cytoskeleton and mitochondria is a key regulator of the behavior of cells [31, 32]. Previous study referred that Wnt5a activation could promote ameloblastoma cell migration [18]. Consistently, we found that Wnt5a up-regulation was linked to significant changes of cellular morphology and increased pseudopod formation number from $11.75 \pm 0.74$ to $14.25 \pm 0.43$ per cell (Figure 4A), and we further found that such exogenous incubation led to significantly increased migration of AM-1 cells (Figure 4B, C). Furthermore, we determined that Wnt5a knockdown inhibited $\mathrm{AB}$ cell migration (Figure 4B, C). Therefore, our results suggest a direct role for Wnt5a in regulating the migratory capacity of these $\mathrm{AB}$ cells.

A Phalloidin
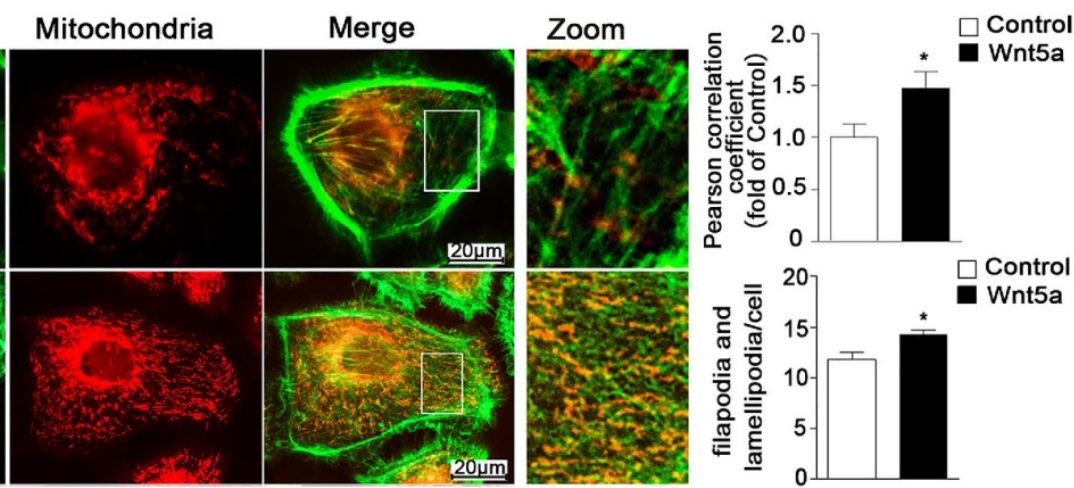

B Control

\begin{abstract}
Wnt5a
\end{abstract}
Scramble

sh-Wnt5a
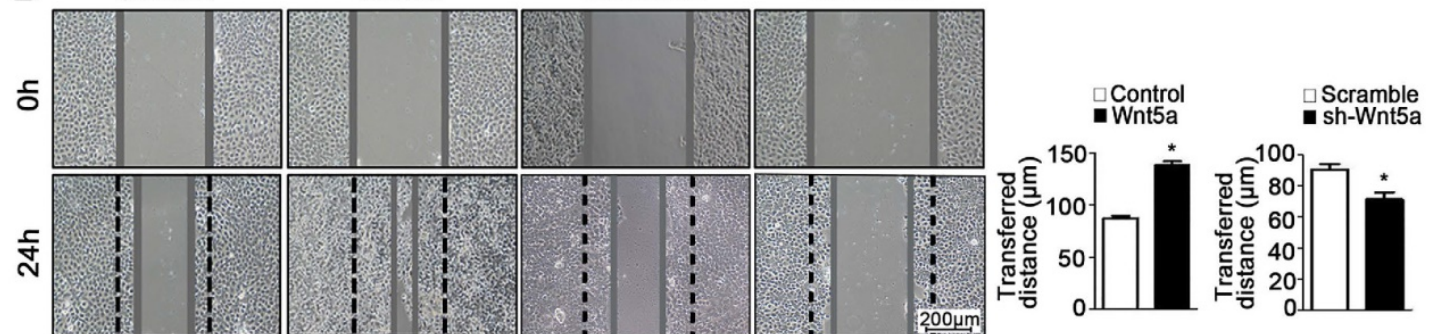

C
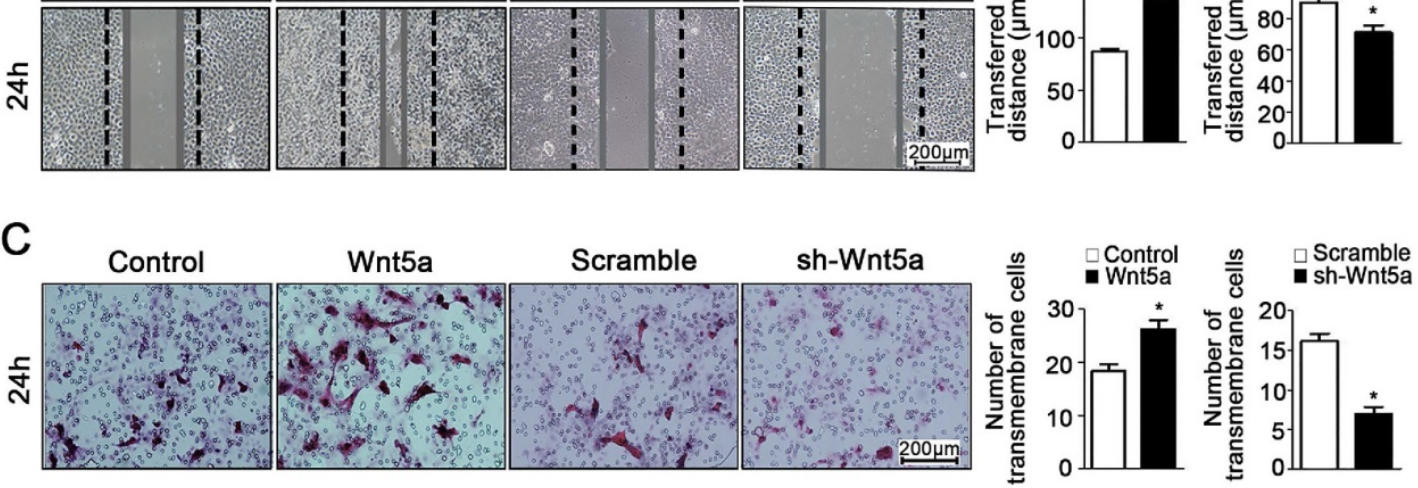

Figure 4. Wnt5a mediates mitochondria-cytoskeleton morphology and migration behavior in AM-1 cells. (A) Confocal images of AM-1 cell culturing in Wnt-5a recombinant protein and then loaded with FITC-phalloidin and Mito Tracker Red. Scale Bar: $20 \mu m$. Green: FITC-phalloidin, Red: Mito Tracker Red. Pearson correlation coefficient quantification of Mitochondria and cytoskeleton co-localization was shown in bar chart (up). N=25-33 frames from 3 independent experiments. The number of filopodia and lamellipodia was counted and calculated in bar chart (down). N=16-20 cells in each group. (B) Scratched at Oh and photographed at 0h, 24h respectively, the transferred distance from Wnt5a up-regulation and down-regulation was calculated and shown in Bar charts respectively. Scale bar: 200 um, $\mathrm{N}=9$ from 3 independent experiments. (C) Transwell assay showing the migration cell numbers of AM-1 cell treated with Wnt5a recombinant protein or sh-Wnt5a adenovirus. Bar charts were the quantitative analysis of up-regulation and down-regulation respectively. Scale bar: $100 \mu \mathrm{m}, \mathrm{N}=5 . *_{\mathrm{p}}<0.05$. 
A
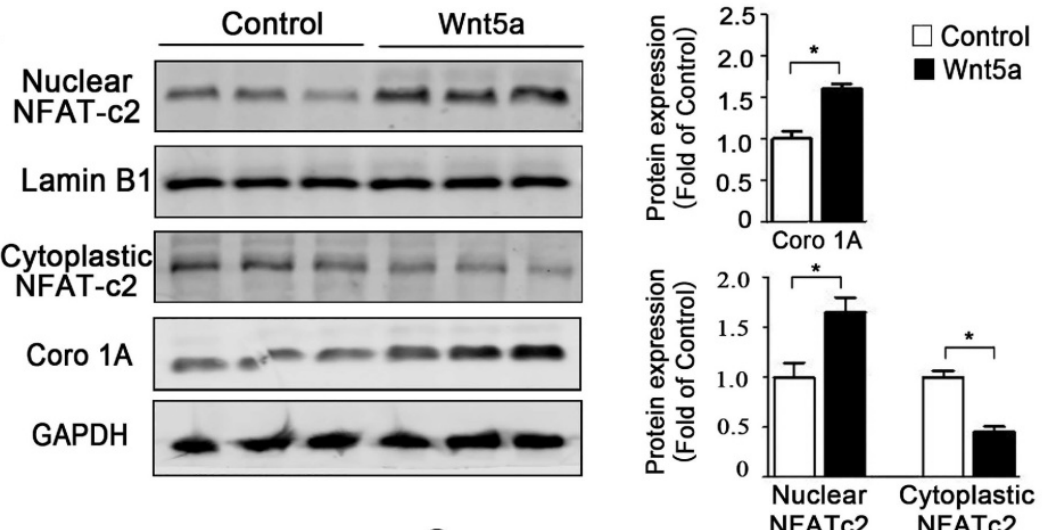

B

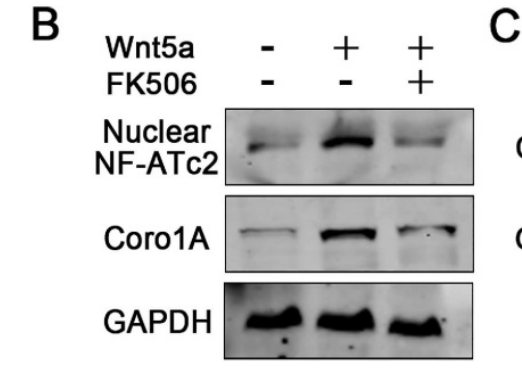

C NFATc2 NFATc2

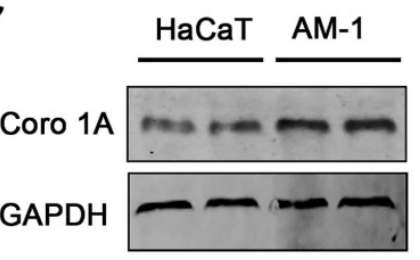

D

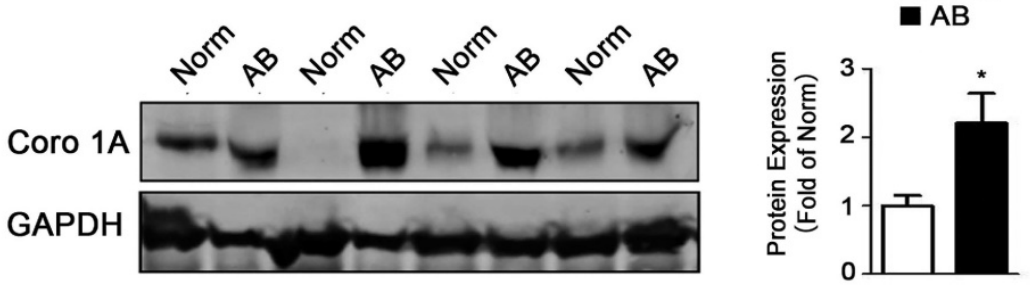

E

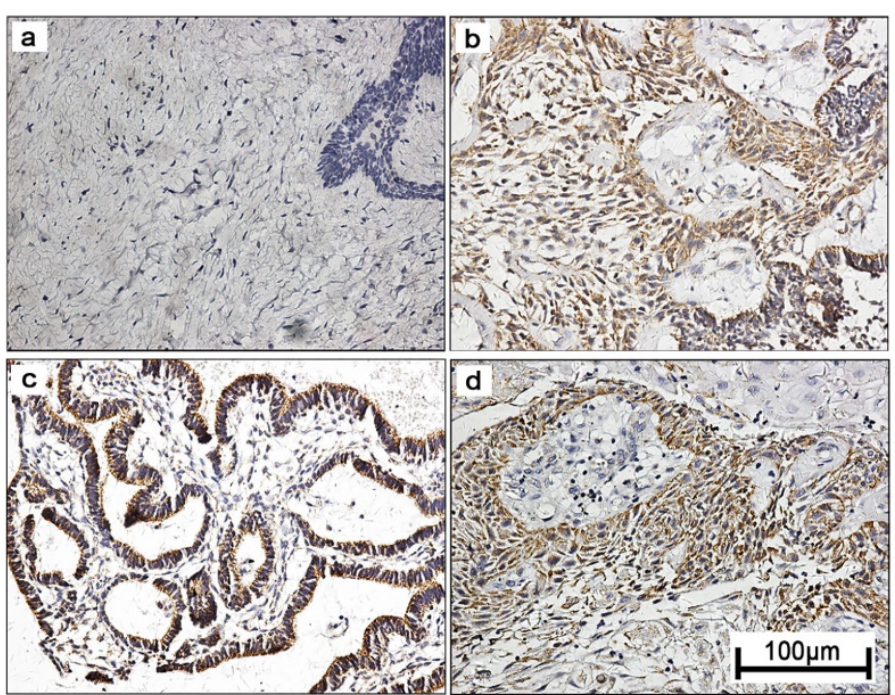

Figure 5. Wnt5a up-regulates mitochondria-cytoskeleton co-localization through mediating Coro I A expression. (A) Western blot detected nuclear transfactor NFATc2 and CorolA protein expression. Bar charts demonstrating quantitative significance results from 3 to 5 independent experiments. (B) Western blot demonstrating Corol A protein expression changes of AM-1 cells treated with only human recombinant Wnt5a compared with cells in the presence of both rhWnt5a and FK506 (200 nm). (C) Western blot showed CorolA protein expression in $\mathrm{HaCaT}$ and AM-1 cells. (D) Western blot results demonstrated CorolA protein expression in tumor adjacent normal tissues and ameloblastoma tissues. Bar charts showed analysis quantification from 8 pairs of tissues. (E) Immunohistochemistry staining demonstrated CorolA protein expression in normal oral mucosa tissues (a), (b-d) ameloblastoma tissues (forlicular type for b, plexiform type for c, peripheral type for d). Magnification: $200 \times$. ${ }^{*}$ < 0.05.

According to the previous studies, Coro1A was considered as an important F-actin binding protein and involved in cytoskeleton dynamic changes. Given this observation, we next sought to determine whether Coro1A played a role in regulating mitochondrial-cytoskeleton interactions or in governing the migration of $\mathrm{AB}$ cells through regulation of the cytoskeletal network. To that end, 
we used adenoviral vectors to generate AM- 1 cells in which Coro1A was knocked down (Figure 6A). Using these cells, it was determined that Coro1A can regulate cytoskeletal dynamics and pseudopod formation (Figure 6B). Moreover, Coro1A knockdown was associated with changes in the mitochondria-cytoskeleton colocalization induced by the addition of recombinant Wnt5a incubation (Figure 6C). Finally, Coro1A knockdown was in association with a significant decrease in the migratory ability of AM-1 cells and could partially overturn Wnt5ainduced increase of migration distance from $124.4 \pm$ $6.44 \mathrm{~nm}$ to $108.3 \pm 5.19 \mathrm{~nm}$ (Figure 6D) and transmembrane cell number from $27.40 \pm 1.63$ to 20.20 \pm 1.36 (Figure 6E). Thus, our results confirm the regulatory role of Coro1A, a Wnt5a downstream targeted protein, on inter-organelle contact networks and $\mathrm{AB}$ biological behavior.
Table 2. Association between CorolA expression and clinical pathological features

\begin{tabular}{|c|c|c|c|c|c|}
\hline Characteristics & $\begin{array}{l}\text { Total } \\
(\mathrm{N})\end{array}$ & $\begin{array}{l}\text { Coro1A } \\
\text { Positive } \\
(\mathrm{N}, \%)\end{array}$ & $\begin{array}{l}\text { Coro1A } \\
\text { Negative } \\
(\mathrm{N}, \%)\end{array}$ & $x^{2}$ & $\mathrm{P}$ \\
\hline Age (year) & & & & 0.080 & 0.777 \\
\hline$\leq 50$ & 69 & $40(57.97)$ & $29(42.03)$ & & \\
\hline$>50$ & 22 & $12(54.55)$ & $10(45.45)$ & & \\
\hline Gender & & & & 0.004 & 0.951 \\
\hline Male & 51 & $29(56.86)$ & $22(43.14)$ & & \\
\hline Female & 40 & $23(57.50)$ & $17(42.50)$ & & \\
\hline Tumor Location & & & & 0.698 & 0.404 \\
\hline Mandible & 80 & $47(58.75)$ & $33(41.25)$ & & \\
\hline Maxilla and Gingiva & 11 & $5(45.45)$ & $6(54.55)$ & & \\
\hline Pathological type & & & & 0.200 & 0.655 \\
\hline Solid/Multi-cystic & 72 & $42(58.33)$ & $30(41.67)$ & & \\
\hline $\begin{array}{l}\text { Others } \\
\text { (Unicystic/Peripheral/Desmoplastic) }\end{array}$ & 19 & $10(52.63)$ & $9(47.37)$ & & \\
\hline Recurrence & & & & 3.857 & $0.0495^{*}$ \\
\hline Yes & 9 & $8(88.89)$ & 1(11.11) & & \\
\hline No & 82 & $45(54.88)$ & $37(45.12)$ & & \\
\hline
\end{tabular}

$\mathrm{P}$ value was determined by Chi-square test. ${ }^{*} \mathrm{P}<0.05$.
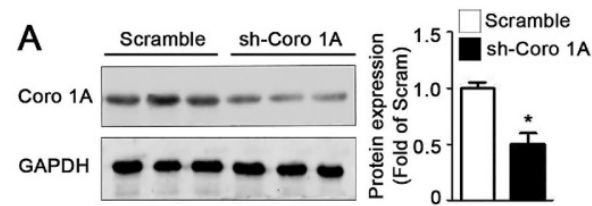

C
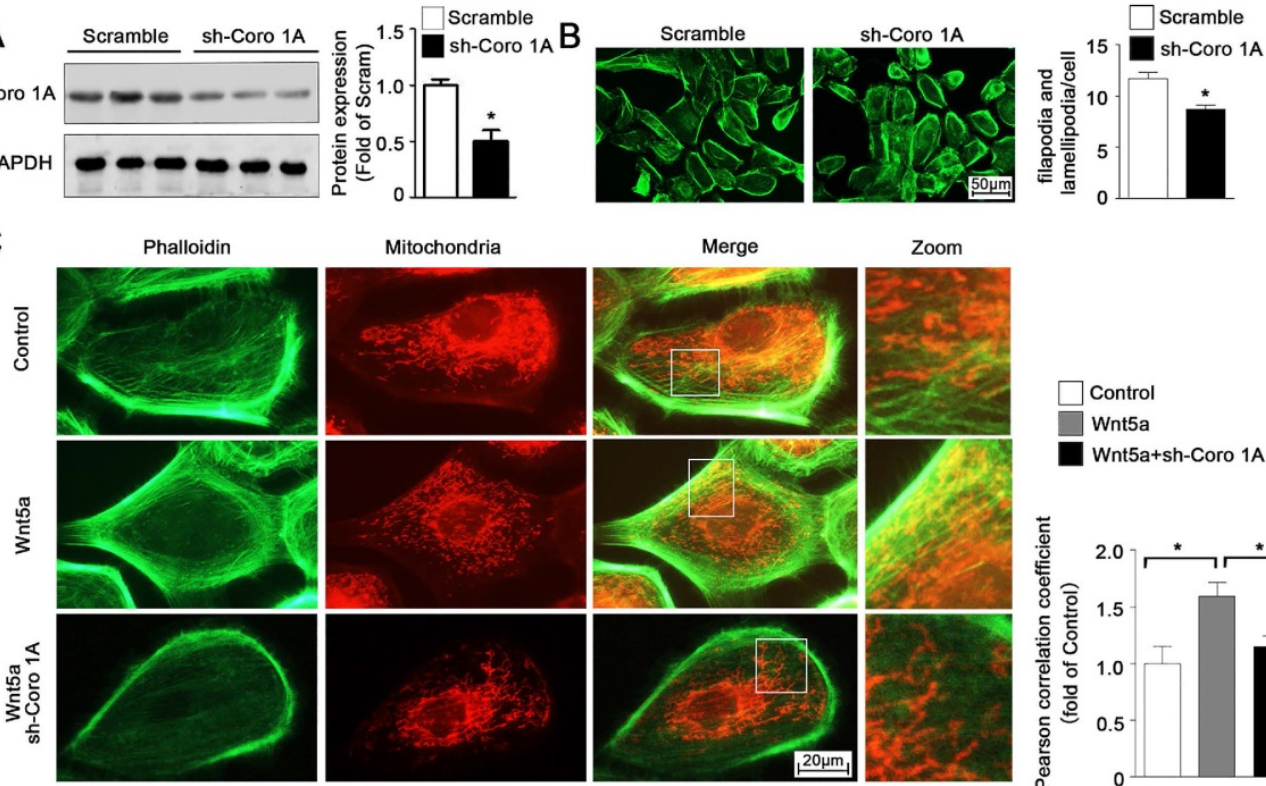

D
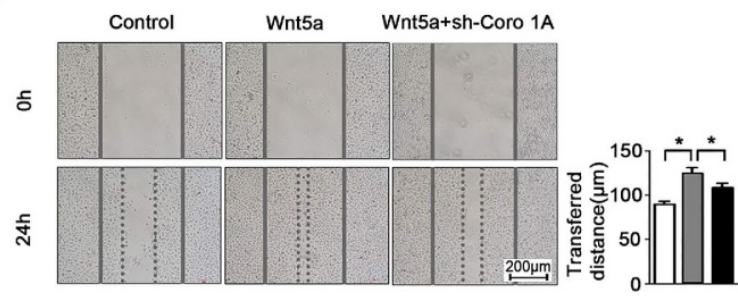

E
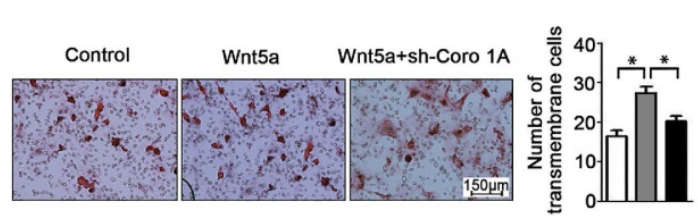

$\square$ Control

Wnt5a

Wnt5a+sh-Coro $1 \mathrm{~A}$

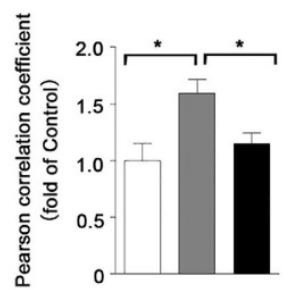

Figure 6. CorolA could change F-actin cytoskeleton dynamics and affect AM-I cell migration. (A) Western blot detecting sh-CorolA adenovirus transfected efficiency. (B) FITC-phalloidin staining showing F-actin and cell morphology changes of both sh-scramble and sh-CorolA adenovirus transfected AM-1 cells. The number of filopodia and lamellipodia was counted and shown in bar charts, $\mathrm{N}=18-26$ cells in each group. (C-E) Sh-scramble and sh-CorolA adenovirus were transfected for $48-72 \mathrm{~h}$, and then Wnt5a recombinant protein was added. Both Mito tracker Red and FITC-philloidin staining demonstrating mitochondria and cytoskeleton contact changes, and pearson correlation coefficient was counted and shown in bar charts, $N=26-43$ frames from 3 independent experiments (C); Scratch (D) and transwell assay (E) showing migration ability changes under corolA decreased expression. $\mathrm{N}=8$ and $\mathrm{N}=5$ independent experiments for scratch and transwell experiments respectively. * $\mathrm{p}<0.05$. 


\section{Discussion}

In the present study, we offered evidence for the central role of Wnt5a in the regulation of the migration of $\mathrm{AB}$ cells owing to its ability to mediate the up-regulation of the F-actin related Coro1A protein and to thereby modulate contact between mitochondria and the cytoskeleton. We found that Wnt5a was significantly up-regulated at the protein level in $A B$ tissue samples relative to normal control tissue samples. In vitro, we further found that Wnt5a up-regulation was associated with increased intracellular calcium levels, in turn leading to changes in mitochondrial dynamics, energy metabolism, and expression of Coro1A. When Wnt5a or Coro1A was knocked down, this reversed these observed changes in intracellular organelle dynamics, and reduced the migratory ability of $\mathrm{AB}$ cells.

Previous studies have revealed the elevated Wnt5a expression in more advanced and aggressive disease in patients with melanoma [33], gastric cancer, lung cancer [34] and prostate cancer [35]. Furthermore, it has also observed that up-regulation of Wnt5a is associated with changes in the epithelialmesenchymal transition within AB cells [18], and ours is the first study to report the role of Wnt5a in regulating mitochondrial-cytoskeletal dynamic changes and thereby regulating $A B$ cell migration. Indeed, we observed that Wnt5a was up-regulated in both $\mathrm{AB}$ tumor tissue samples and AM-1 cells, consistent with its potential role in the development and progression of $\mathrm{AB}$. The molecular basis for Wnt5a up-regulation in $\mathrm{AB}$ will require further research, but may be associated with changes in the expression of certain transcription factors or microRNAs controlling Wnt5a expression [36, 37]. Future studies regarding the control of Wnt5a expression in $\mathrm{AB}$ and other tumors will be invaluable, as they may offer insight into how to target the Wnt5a signaling pathway to effectively treat these tumors.

The endoplasmic reticulum (ER) serves as a primary calcium storage site within the cell, and can thus release $\mathrm{Ca}^{2+}$ into the cytoplasm in specific contexts, resulting in increased local mitochondrial calcium concentrations owing to the close structural interactions between mitochondria and the ER that initiate mitochondrial $\mathrm{Ca}^{2+}$ uptake [38, 39]. Increases in concentrations of $\mathrm{Ca}^{2+}$ within mitochondria can in turn lead to TCA cycle activation and oxidative phosphorylation, thereby enhancing mitochondrial energy production within the cell [25, 40, 41]. Importantly, previous work has demonstrated that elevated uptake of $\mathrm{Ca}^{2+}$ by mitochondria is frequently associated with the enhanced migration and invasive activity of tumor cells. In this study, we found that Wnt5a can drive elevated intracellular and mitochondrial calcium levels and ATP synthesis, thus potentially providing more energy to facilitate $\mathrm{AB}$ cell migration. Elevated mitochondrial $\mathrm{Ca}^{2+}$ levels can also drive increased calcium-sensitive dehydrogenase and $\mathrm{NADH}$ activity, driving electron flow through the respiratory chain and thereby further increasing ATP synthesis [41]. We hypothesized that Wnt5a signaling pathway could result in the elevation of mitochondrial calcium levels within $\mathrm{AB}$ cells, thereby driving the enhanced activity of calcium-sensitive enzymes in the respiratory chain and consequently increasing energy production within these tumor cells, thus facilitating their migration ability.

In the present study, we observed changes in the dynamics of mitochondria. A number of different proteins may regulate the fusion and division of mitochondria within cells [42]. Our results suggested the possibility that these observed changes in mitochondrial morphology in AB cells may be closely linked to mitochondrial $\mathrm{Ca}^{2+}$ homeostasis changes caused by Wnt5a. Such altered calcium homeostasis would in turn could activate PKC signaling and thereby mediate the phosphorylation of fissionassociated proteins, thus modulating mitochondrial dynamics [43]. The cytoskeleton is a key regulator of the dynamic rearrangement of organelles within cells, allowing for deformation, mitochondrial transport, signaling, and cell motility [44, 45]. In this study, we found that Wnt5a could function as a regulator of the rearrangement of this mitochondrial-cytoskeletal network, thereby potentially regulating $\mathrm{AB}$ cell migratory activity.

Intracellular calcium signaling can, in addition to the activities outlined above, result in the activation of calcium-sensitive transcription factors [46]. These include transcription factors in the NFAT family, which consists of 5 family members, of which four (NFAT1-4) are known to be regulated by $\mathrm{Ca}^{2+}$ signaling [47]. In the present study, we observed significant NFAT1 activation and nuclear localization in the context of Wnt5a up-regulation, in turn regulating the expression of the downstream target gene encoding Coro1A. Coro1A protein was first shown to be involved in the migratory activity and morphological changes observed in T cells [48, 49]. More recent studies have shown Coro1A to be a key F-actin binding protein important for the control of cytoskeletal dynamics in myriad contexts [50,51]. We found that in $\mathrm{AB}$ cells, Coro1A regulated cytoskeletal dynamics and interactions between mitochondria and cytoskeleton, thereby regulating the migration of these tumor cells. When Coro1A was knocked down, $\mathrm{AB}$ cell migration was significantly reduced, thus identifying Coro1A as a potentially viable therapeutic target for $A B$ treatment. Our results showed that 
Wnt5a could regulate $\mathrm{Ca}^{2+}$ pathway and activate downstream target proteins. However, previous studies also have found that Wnt5a was involved in PCP pathway, which was recognized as an important signaling pathway to regulate tissue polarity and cell migration [52, 53]. Since Wnt5a can still promote cell migration in Coro1A-knockdown cells, we hypothesized that other migration related pathways could also be activated by Wnt5a, which still need further investigation.

\section{Conclusion}

Our findings demonstrated the increased Wnt5a expression in $A B$, and its up-regulation enhanced $A B$ cell migration capacity through increasing intracellular calcium levels, in turn leading to changes in mitochondrial dynamics, energy metabolism, and expression of Coro1A. Knockdown of Wnt5a or downstream Coro1A could reverse these observed changes in intracellular organelle dynamics, and reduce the migratory ability of $\mathrm{AB}$ cells. Thus, our findings revealed the role of $\mathrm{Wnt} 5 \mathrm{a}$ and Coro $1 \mathrm{~A}$ in $\mathrm{AB}$ for the first time, and provided new therapeutic targets for disease progression.

\section{Abbreviations}

AB: ameloblastoma; NOM: normal oral mucosa; TEM: transmission electron microscopy; Coro1A: Coronin-1A; ER: endoplasmic reticulum.

\section{Acknowledgements}

Thanks to Professor Toshinari Mikami of Iwate Medical University for providing the cell line.

\section{Funding}

This work was funded by the National Natural Science Foundation of China (81072197 and 81470758).

\section{Availability of data and material}

The datasets analyzed during the current study are available from the corresponding author on reasonable request.

\section{Author Contributions}

Ming Zhong conceived and designed the study. Xue Qiao, Xing Niu and Junxiu Shi conducted most of the experiments and data analysis, and wrote the manuscript. Lijie Chen, Xiaobin Wang, Jinwen Liu and Li Zhu participated in collecting data and helped to draft the manuscript. All authors reviewed and approved the manuscript.

\section{Ethics approval and consent to participate}

The study was approved by the Ethics Committee of School and Hospital of Stomatology, China Medical University (2016-12).

\section{Consent for publication}

All subjects were informed consent.

\section{Competing Interests}

The authors have declared that no competing interest exists.

\section{References}

1. Kondo S, Ota A, Ono T, Karnan S, Wahiduzzaman M, Hyodo T, et al. Discovery of novel molecular characteristics and cellular biological properties in ameloblastoma. Cancer Med. 2020; 9: 2904-17.

2. Sun Y, Niu X, Wang G, Oiao X, Chen L, Zhong M. A Novel IncRNA ENST00000512916 Facilitates Cell Proliferation, Migration and Cell Cycle Progression in Ameloblastoma. Onco Targets Ther. 2020; 13: 1519-31.

3. Wright JM, Vered M. Update from the 4th Edition of the World Health Organization Classification of Head and Neck Tumours: Odontogenic and Maxillofacial Bone Tumors. Head Neck Pathol. 2017; 11: 68-77.

4. Speight PM, Takata T. New tumour entities in the 4th edition of the World Health Organization Classification of Head and Neck tumours: odontogenic and maxillofacial bone tumours. Virchows Archiv : an international journal of pathology. 2018; 472: 331-9.

5. McClary AC, West RB, McClary AC, Pollack JR, Fischbein NJ, Holsinger CF, et al. Ameloblastoma: a clinical review and trends in management. European archives of oto-rhino-laryngology : official journal of the European Federation of Oto-Rhino-Laryngological Societies (EUFOS) : affiliated with the German Society for Oto-Rhino-Laryngology - Head and Neck Surgery. 2016; 273: 1649-61.

6. Abiko Y, Nagayasu H, Takeshima M, Yamazaki M, Nishimura M, Kusano K, et al. Ameloblastic carcinoma ex ameloblastoma: report of a case-possible involvement of $\mathrm{CpG}$ island hypermethylation of the p16 gene in malignant transformation. Oral surgery, oral medicine, oral pathology, oral radiology, and endodontics. 2007; 103: 72-6.

7. Lin $Y, \mathrm{He} \mathrm{JF}, \mathrm{Li} \mathrm{ZY}$, Liu JH. Ameloblastoma with varied sites of metastasis: report of two cases and literature review. Journal of cranio-maxillo-facial surgery : official publication of the European Association for Cranio-Maxillo-Facial Surgery. 2014; 42: e301-4.

8. Jose C, Bellance N, Rossignol R. Choosing between glycolysis and oxidative phosphorylation: A tumor's dilemma? Bba-Bioenergetics. 2011; 1807: 552-61.

9. Liberti MV, Locasale JW. The Warburg Effect: How Does it Benefit Cancer Cells? Trends in biochemical sciences. 2016; 41: 211-8.

10. Desai SP, Bhatia SN, Toner M, Irimia D. Mitochondrial Localization and the Persistent Migration of Epithelial Cancer cells. Biophysical journal. 2013; 104: 2077-88.

11. Altieri DC. Mitochondria on the move: emerging paradigms of organelle trafficking in tumour plasticity and metastasis. British journal of cancer. 2017; 117: 301-5.

12. A M, Fung TS, Kettenbach AN, Chakrabarti R, Higgs HN. A complex containing lysine-acetylated actin inhibits the formin INF2. Nature cell biology. 2019; 21: 592-602.

13. Maes H, Van Eygen S, Krysko DV, Vandenabeele P, Nys K, Rillaerts K, et al. BNIP3 supports melanoma cell migration and vasculogenic mimicry by orchestrating the actin cytoskeleton. Cell death \& disease. 2014; 5: e1127.

14. Tran FH, Zheng JJ. Modulating the wnt signaling pathway with small molecules. Protein science : a publication of the Protein Society. 2017; 26: 650-61.

15. Asem MS, Buechler S, Wates RB, Miller DL, Stack MS. Wnt5a Signaling in Cancer. Cancers. 2016; 8: 79.

16. Sakamoto T, Kawano S, Matsubara R, Goto Y, Jinno T, Maruse Y, et al. Critical roles of Wnt5a-Ror2 signaling in aggressiveness of tongue squamous cell carcinoma and production of matrix metalloproteinase-2 via DeltaNp63beta-mediated epithelial-mesenchymal transition. Oral oncology. 2017; 69: 15-25.

17. Prgomet Z, Andersson T, Lindberg P. Higher expression of WNT5A protein in oral squamous cell carcinoma compared with dysplasia and oral mucosa with a normal appearance. European journal of oral sciences. 2017; 125: 237-46.

18. Sukarawan W, Simmons D, Suggs C, Long K, Wright JT. WNT5A expression in ameloblastoma and its roles in regulating enamel epithelium tumorigenic behaviors. The American journal of pathology. 2010; 176: 461-71.

19. Yoon JC, Ng A, Kim BH, Bianco A, Xavier RJ, Elledge SJ. Wnt signaling regulates mitochondrial physiology and insulin sensitivity. Genes \& development. 2010; 24: 1507-18.

20. Brown K, Yang P, Salvador D, Kulikauskas R, Ruohola-Baker H, Robitaille $\mathrm{AM}$, et al. WNT/beta-catenin signaling regulates mitochondrial activity to alter the oncogenic potential of melanoma in a PTEN-dependent manner. Oncogene. 2017; 36: 3119-36.

21. Brown NA, Rolland D, McHugh JB, Weigelin HC, Zhao L, Lim MS, et al. Activating FGFR2-RAS-BRAF mutations in ameloblastoma. Clin Cancer Res. 2014; 20: 5517-26. 
22. Cong W, Liu B, Liu S, Sun M, Liu H, Yang Y, et al. Implications of the Wnt5a/CaMKII pathway in retinoic acid-induced myogenic tongue abnormalities of developing mice. Scientific reports. 2014; 4: 6082.

23. Ando F, Sohara E, Morimoto T, Yui N, Nomura N, Kikuchi E, et al. Wnt5a induces renal AQP2 expression by activating calcineurin signalling pathway. Nature communications. 2016; 7: 13636.

24. Koopmans T, Kumawat K, Halayko AJ, Gosens R. Regulation of actin dynamics by WNT-5A: implications for human airway smooth muscle contraction. Scientific reports. 2016; 6: 30676

25. Giorgi C, Marchi S, Pinton P. The machineries, regulation and cellular functions of mitochondrial calcium. Nature reviews Molecular cell biology. 2018; 19: 713-30.

26. Moore AS, Holzbaur ELF. Mitochondrial-cytoskeletal interactions: dynamic associations that facilitate network function and remodeling. Current opinion in physiology. 2018; 3: 94-100.

27. Lawrence EJ, Boucher E, Mandato CA. Mitochondria-cytoskeleton associations in mammalian cytokinesis. Cell division. 2016; 11: 3

28. Melkov A, Abdu U. Regulation of long-distance transport of mitochondria along microtubules. Cellular and molecular life sciences : CMLS. 2018; 75: 163-76.

29. Mehta K, Chacko LA, Chug MK, Jhunjhunwala S, Ananthanarayanan V. Association of mitochondria with microtubules inhibits mitochondrial fission by precluding assembly of the fission protein Dnm1. The Journal of biological chemistry. 2019; 294: 3385-96.

30. Zhao Q, Ye M, Yang W, Wang M, Li M, Gu C, et al. Effect of Mst1 on Endometriosis Apoptosis and Migration: Role of Drp1-Related Mitochondrial Fission and Parkin-Required Mitophagy. Cellular physiology and biochemistry : international journal of experimental cellular physiology, biochemistry, and pharmacology. 2018; 45: 1172-90

31. Bartolak-Suki E, Imsirovic J, Nishibori Y, Krishnan R, Suki B. Regulation of Mitochondrial Structure and Dynamics by the Cytoskeleton and Mechanical Factors. International journal of molecular sciences. 2017; 18: 1812.

32. Mogessie B, Zenner H, Renkawitz J. Meeting report - Cell dynamics: organelle-cytoskeleton interface. Journal of cell science. 2019; 132: jcs236679.

33. Weeraratna AT, Jiang Y, Hostetter G, Rosenblatt K, Duray P, Bittner M, et al. Wnt5a signaling directly affects cell motility and invasion of metastatic melanoma. Cancer cell. 2002; 1: 279-88.

34. Huang CL, Liu D, Nakano J, Ishikawa S, Kontani K, Yokomise H, et al. Wnt5a expression is associated with the tumor proliferation and the stromal vascular endothelial growth factor--an expression in non-small-cell lung cancer. Journal of clinical oncology : official journal of the American Society of Clinical Oncology. 2005; 23: 8765-73.

35. Wang Q, Williamson M, Bott S, Brookman-Amissah N, Freeman A, Nariculam J, et al. Hypomethylation of WNT5A, CRIP1 and S100P in prostate cancer. Oncogene. 2007; 26: 6560-5.

36. Shi D, Wang H, Ding M, Yang M, Li C, Yang W, et al. MicroRNA-26a-5p inhibits proliferation, invasion and metastasis by repressing the expression of Wnt5a in papillary thyroid carcinoma. OncoTargets and therapy. 2019; 12: 6605-16.

37. Zhang KK, Geng YD, Wang SB, Huo L. [MicroRNA-26a-5p targets Wnt5a to regulate osteogenic differentiation of human periodontal ligament stem cell from inflammatory microenvironment]. Zhonghua kou qiang yi xue za zhi $=$ Zhonghua kouqiang yixue zazhi $=$ Chinese journal of stomatology. 2019; 54: 662-9.

38. Qiao $X$, Jia $S$, Ye J, Fang $X$, Zhang $C$, Cao $Y$, et al. PTPIP51 regulates mouse cardiac ischemia/reperfusion through mediating the mitochondria-SR junction. Scientific reports. 2017; 7: 45379.

39. Rowland AA, Voeltz GK. Endoplasmic reticulum-mitochondria contacts: function of the junction. Nature reviews Molecular cell biology. 2012; 13: 607-25.

40. Fink BD, Bai F, Yu L, Sivitz WI. Regulation of ATP production: dependence on calcium concentration and respiratory state. American journal of physiology Cell physiology. 2017; 313: C146-C153.

41. Rizzuto R, De Stefani D, Raffaello A, Mammucari C. Mitochondria as sensors and regulators of calcium signalling. Nature reviews Molecular cell biology. 2012; 13: 566-78.

42. Westermann B. Mitochondrial fusion and fission in cell life and death. Nature reviews Molecular cell biology. 2010; 11: 872-84.

43. Dodd AN, Kudla J, Sanders D. The language of calcium signaling. Annual review of plant biology. 2010; 61: 593-620.

44. Fletcher DA, Mullins RD. Cell mechanics and the cytoskeleton. Nature. 2010; 463: 485-92.

45. Yamaguchi H, Condeelis J. Regulation of the actin cytoskeleton in cancer cell migration and invasion. Biochimica et biophysica acta. 2007; 1773: 642-52.

46. Puri BK. Calcium Signaling and Gene Expression. Advances in experimental medicine and biology. 2020; 1131: 537-45.

47. Muller MR, Rao A. NFAT, immunity and cancer: a transcription factor comes of age. Nature reviews Immunology. 2010; 10: 645-56.

48. Mueller P, Massner J, Jayachandran R, Combaluzier B, Albrecht I, Gatfield J, et al. Regulation of $\mathrm{T}$ cell survival through coronin-1-mediated generation of inositol-1,4,5-trisphosphate and calcium mobilization after $\mathrm{T}$ cell receptor triggering. Nature immunology. 2008; 9: 424-31.

49. Stocker TJ, Pircher J, Skenderi A, Ehrlich A, Eberle C, Megens RTA, et al. The Actin Regulator Coronin-1A Modulates Platelet Shape Change and
Consolidates Arterial Thrombosis. Thrombosis and haemostasis. 2018; 118: 2098-111.

50. Castro-Castro A, Ojeda V, Barreira M, Sauzeau V, Navarro-Lerida I, Muriel O, et al. Coronin $1 \mathrm{~A}$ promotes a cytoskeletal-based feedback loop that facilitates Rac1 translocation and activation. The EMBO journal. 2011; 30: 3913-27.

51. Mugnier B, Nal B, Verthuy C, Boyer C, Lam D, Chasson L, et al. Coronin-1A links cytoskeleton dynamics to TCR alpha beta-induced cell signaling. PloS one. 2008 ; 3: e3467.

52. Kikuchi A, Yamamoto H, Sato A, Matsumoto S. Wnt5a: its signalling, functions and implication in diseases. Acta physiologica. 2012; 204: 17-33.

53. Martineau X, Abed E, Martel-Pelletier J, Pelletier JP, Lajeunesse D. Alteration of Wnt5a expression and of the non-canonical Wnt/PCP and Wnt/PKC-Ca2+ pathways in human osteoarthritis osteoblasts. PloS one. 2017; 12: e0180711. 